\title{
Máscara laríngea vs tubo orotraqueal no atendimento pré-hospitalar - desfechos hospitalares
}

\author{
Laringeal mask vs orotraqueal tube in pre-hospital \\ care - hospital outcomes
}

\author{
Renata Bernardy Prestes' ${ }^{1}$, Paula Caitano Fontela², \\ William Maia Coutinho ${ }^{3}$, Luiz Alberto Forgiarini Junior ${ }^{4}$
}

1'Formação em Negócios e Saúde. Santa Cruz do Sul, Rio Grande do Sul, Brasil. ORCID: 0000-0002-7297-1645. rebernardy@yahoo.com.br ${ }^{2}$ Hospital de Caridade de ljuí. ljuí, Rio Grande do Sul, Brasil. ORCID: 0000-0003-0086-7037. paula_fontella@hotmail.com ${ }^{3}$ Hospital Independência - Rede Divina Providência. Porto Alegre, Rio Grande do Sul, Brasil. ORCID: 0000-0001-5201-5018. coutinho.william89@gmail.com ${ }^{4}$ Autor para correspondência. Universidade La Salle, Faculdade Inspirar. Canoas, Rio Grande do Sul, Brasil. ORCID: 0000-0002-6706-2703. forgiarini.luiz@gmail.com

RESUMO | INTRODUÇÃO: Em situações emergenciais, se torna crucial o manejo adequado das vias aéreas, pois falhas neste processo contribuem consideravelmente para piores desfechos clínicos. OBJETIVO: comparar a utilização do tubo endotraqueal e da máscara laríngea no atendimento pré-hospitalar em relação aos desfechos hospitalares tais como tempo de ventilação mecânica, tempo de permanência na UTI e taxa de óbito. MATERIAIS E MÉTODOS: estudo observacional retrospectivo, desenvolvido no Hospital Santa Cruz, Rio Grande do Sul. Foram incluídos pacientes maiores de 18 anos, de ambos os sexos, que foram atendidos pelo SAMU durante o atendimento pré-hospitalar, e após o encaminhados para o Pronto Atendimento ou Ambulatório do Hospital Santa Cruz. As variáveis analisadas foram extraídas dos prontuários médicos dos indivíduos selecionados. RESULTADOS: foram analisados 27 indivíduos, maioria do sexo masculino, com média de idade de $46,5 \pm 22$ anos, sendo a causa predominante do atendimento pré-hospitalar o politraumatismo. Ao compararmos os pacientes que utilizaram tubo endotraqueal com os que utilizaram máscara laríngea, não foram observadas diferenças significativas em relação ao tempo de atendimento pré-hospitalar. Na fase intra-hospitalar, observamos diferença significativa em relação ao tempo de ventilação mecânica e tempo de internação na UTI, não sendo o mesmo observado em relação a taxa de óbito. CONCLUSÃo: Os pacientes submetidos a utilização da máscara laríngea no atendimento pré-hospitalar apresentaram menor tempo de ventilação mecânica e menor tempo de internação na UTI, em relação aos pacientes intubados com tubo endotraqueal.

PALAVRAS-CHAVE: Serviços médicos de emergência. Intubação intratraqueal. Máscaras laríngeas. Respiração artificial. Avaliação de resultados (cuidados de saúde).
ABSTRACT | INTRODUCTION: In emergency situations, proper management of the airways becomes crucial, because flaws in this process contribute considerably to worse clinical outcomes. OBJECTIVE: To compare the use of the endotracheal tube and laryngeal mask in prehospital care in relation to hospital outcomes such as mechanical ventilation time, length of stay in the ICU and death rate. MATERIALS AND METHODS: a retrospective observational study, developed in a Hospital Santa Cruz, Rio Grande do Sul. Patient records were used from the Emergency Mobile Service (SAMU) of Santa Cruz do Sul, sent to the Emergency Department or Ambulatory of Santa Cruz Hospital. RESULTS: 27 individuals, mostly males, with an average age of $46.5 \pm 22$ years, were analyzed, being the predominant cause of pre-hospital care the polytrauma. When comparing the patients who used the endotracheal tube with those who used laryngeal mask, no significant differences were observed in relation to the time of prehospital care. In the in-hospital phase, we observed a significant difference in relation to the time of mechanical ventilation and length of stay in the ICU, and the same was not observed in relation to the death rate. CONCLUSION: The patients submitted to the use of the laryngeal mask in the pre-hospital care presented a shorter time of mechanical ventilation and a shorter time of hospitalization in the ICU than the patients intubated with an endotracheal tube.

KEYWORDS: Emergency medical services. Intratracheal intubation. Laryngeal masks. Artificial respiration. Evaluation of results (health care). 


\section{Introdução}

O atendimento pré-hospitalar configura-se como uma modalidade de assistência que objetiva atender precocemente às vítimas e ainda prestar o transporte adequado para um serviço de saúde integrado, visando reduzir o agravamento das lesões e, consequentemente, os níveis de morbidade e mortalidade ${ }^{1}$.

Os principais benefícios do atendimento pré-hospitalar são realizados durante a segunda fase do trauma, quando a prestação de cuidados pode limitar ou interromper a cascata de eventos que podem levar à morte ou incapacidade ao longo da vida. Sem este cuidado, pessoas que poderiam sobreviver aos seus ferimentos, podem vir a morrer no local ou a caminho do hospital. A maioria das mortes nas primeiras horas após a lesão é o resultado de comprometimentos das vias aéreas, insuficiência respiratória, bem como hemorragias não controladas².

Neste contexto, então, se torna crucial o manejo adequado das vias aéreas, pois falhas neste processo contribuem consideravelmente para piores desfechos clínicos ${ }^{3}$. Existem várias recomendações nos casos de insucesso na intubação endotraqueal, dentre elas a utilização os dispositivos supraglóticos, como a máscara laríngea, que se apresentam como uma boa alternativa e estão na primeira linha de escolha nessas situações ${ }^{4,5}$.

A máscara laríngea consiste em um tubo semelhante ao tubo endotraqueal, com uma máscara inflável na extremidade distal, apropriada para adaptação à faringe posterior, selando a região da base da língua e da abertura laríngea ${ }^{6-9}$. A facilidade de manuseio desse dispositivo o torna útil no estabelecimento de ventilação em pacientes em situação de risco, principalmente entre os profissionais pouco treinados para a intubação endotraqueal ${ }^{6,9-12}$.

Sendo assim, o objetivo deste estudo foi comparar os desfechos como tempo de ventilação mecânica, tempo de permanência hospitalar e óbito entre os pacientes que utilizaram tubo endotraqueal e a máscara laríngea durante o atendimento pré-hospitalar.

\section{Materiais e Métodos}

Trata-se de um estudo observacional retrospectivo, desenvolvido no Hospital Santa Cruz na cidade de Santa Cruz/RS. Foram revisados os prontuários de pacientes provenientes do Serviço de Atendimento Móvel de Urgência (SAMU) de Santa Cruz do Sul que necessitaram de intubação traqueal e que foram encaminhados para este hospital durante o período de março de 2014 a dezembro de 2015. O presente estudo foi aprovado pelo Comitê de Ética em Pesquisa do Centro Universitário Metodista (IPA), com parecer $n^{\circ}$ 1.374.978 (CAAE 49630315.3.0000.5308).

Foram incluídos pacientes maiores de 18 anos, de ambos os sexos, que foram atendidos pelo SAMU e intubados durante o atendimento pré-hospitalar, seja com tubo endotraqueal ou máscara laríngea. Foram excluídos os pacientes que ingressaram no hospital com óbito previamente constatado.

Foram avaliados a partir dos prontuários dos atendimento pré-hospitalar a causa do atendimento, o tempo deste e o dispositivo utilizado para a intubação traqueal. Durante o período de internação hospitalar avaliamos a permanência destes pacientes na emergência e/ou unidade de terapia intensiva (UTI), tempo de ventilação mecânica, complicações durante a internação,transferência hospitalar e mortalidade. Todos os dados contínuos foram descritos através de média e desvio-padrão ou mediana (mínimo - máximo) e os categóricos através de frequência absoluta e percentual. A normalidade foi aferida através do teste de Shapiro-Wilk. A comparação entre os grupos dos pacientes que foram intubados com o tubo endotraqueal e os pacientes que se utilizou a máscara laríngea foi realizada através do teste $t$ de Student Teste $U$ de Mann-Whitney. Todos os dados foram armazenados e analisados no software Statistical Package for the Social Sciences (SPSS) for Windows 20.0, sendo adotado um nível de significância de 5\%. 


\section{Resultados}

Durante o período do estudo, 27 indivíduos atenderam os critérios de elegibilidade do estudo. A média de idade foi de $46,5 \pm 22$ anos, houve prevalência do sexo masculino $(67,8 \%)$ e o atendimento pré-hospitalar mais observada foi o politraumatismo, (Tabela 1).

Os pacientes intubados com a máscara laríngea totalizaram 13, o tempo de atendimento pré-hospitalar foi de $24,14 \pm 8,19$ minutos. O tempo de internação na UTI e de ventilação mecânica foram de 3,5 $(1$ - 11) dias e 1 (1 - 5) dias, respectivamente. Destes, sete foram encaminhados para UTI, com dois evoluindo para óbito. Em relação aos demais, um indivíduo foi internado na unidade de enfermaria, dois foram transferidos para outro hospital e três evoluíram para óbito.
Quatorze pacientes foram intubados com tubo endotraqueal no atendimento pré-hospitalar, o tempo deste atendimento foi de 29,13 $\pm 15,48$ minutos, $O$ tempo de internação na UTI e o tempo de ventilação mecânica foram de 7 (3 - 18) dias e $5(1$ - 12) dias, respectivamente. Deste grupo, oito pacientes foram encaminhados para a UTI, dos quais três evoluíram para óbito. Os demais pacientes foram internados na emergência do hospital, com quatro destes evoluindo para óbito. (Tabela 2).

Quando comparados os indivíduos que utilizaram máscara laríngea e tubo endotraqueal para a intubação traqueal, não foram observadas diferenças significativas em relação ao tempo de atendimento pré-hospitalar. Já na fase de atendimento hospitalar, observamos menor tempo de ventilação mecânica ( $p=$ $0,03)$ e menor tempo de internação na UTI $(p=0,004)$ nos pacientes submetidos a intubação com a máscara laríngea. Em relação a taxa de óbito, não foi observada diferença significativa, entre os grupos (Tabela 2).

Tabela 1. Características dos pacientes incluídos no estudo relacionado a intubação pré-hospitalar, ano 2015, Santa Cruz/RS. ( $n=27$ )

\begin{tabular}{|c|c|}
\hline Variáveis & Resultado \\
\hline Idade, anos & $46,5 \pm 22$ \\
\hline \multicolumn{2}{|l|}{ Sexo } \\
\hline Masculino & $19(67,8 \%)$ \\
\hline Feminino & $8(32,1 \%)$ \\
\hline \multicolumn{2}{|l|}{ Causa do atendimento pré-hospitalar } \\
\hline Politraumatismo & $10(35,7 \%)$ \\
\hline Neurológico & $5(17,8 \%)$ \\
\hline Outros & $12(42,8 \%)$ \\
\hline Tempo de atendimento pré-hospitalar, min & $13,5 \pm 5,4$ \\
\hline \multicolumn{2}{|l|}{ Via aérea artificial utilizada } \\
\hline Máscara laríngea & $13(46,4 \%)$ \\
\hline Tubo endotraqueal & $14(53,6 \%)$ \\
\hline
\end{tabular}

Dados expressos em médias \pm desvio padrão e $n$ (\%). Min - minutos. 
Tabela 2. Comparação entre os pacientes intubados com máscara laríngea e tubo endotraqueal, ano 2015, Santa Cruz/RS

\begin{tabular}{|c|c|c|c|}
\hline Variáveis & $\begin{array}{l}\text { Máscara } \\
\text { Larígea }\end{array}$ & $\begin{array}{c}\text { Tubo } \\
\text { Endotraqueal }\end{array}$ & $\mathbf{P}$ \\
\hline Idade, anos & $44,54 \pm 24,31$ & $47,29 \pm 20,11$ & 0,652 \\
\hline \multicolumn{4}{|l|}{ Sexo, n (\%) } \\
\hline Masculino & $10(77,5)$ & $9(64,3)$ & 0,721 \\
\hline Feminino & $3(22,5)$ & $5(35,7)$ & 0,598 \\
\hline \multicolumn{4}{|l|}{ Causa do atendimento, $\mathrm{n}(\%)$} \\
\hline Politraumatismo & $6(46,1)$ & $5(35,7)$ & \\
\hline IRA & $2(15,4)$ & $3(21,4)$ & \\
\hline FAF & $2(15,4)$ & $2(14,3)$ & \\
\hline PCR & $1(7,7)$ & $4(28,6)$ & \\
\hline AVC & $1(7,7)$ & - & \\
\hline \multirow[t]{2}{*}{ FAB } & $1(7,7)$ & - & \\
\hline & & $29,13 \pm 15,48$ & 0,141 \\
\hline Tempo de atendimento pré-hospitalar, min & $24,14 \pm 8,19$ & & \\
\hline Tempo de internação na UTI, dias & $3,5(1-11)$ & $7(3-18)$ & 0,004 \\
\hline Tempo de ventilação mecânica, dias & $1(1-5)$ & $5(1-12)$ & 0,03 \\
\hline \multicolumn{4}{|l|}{ Complicações, n (\%) } \\
\hline Infecção respiratória & $2(15,4)$ & $4(28,6)$ & \\
\hline Lesão de faringe & $1(7,7)$ & - & \\
\hline Transferência hospitalar, n (\%) & $2(15,4)$ & 0 & \\
\hline Internação no andar, n (\%) & $1(7,7)$ & $2(14,3)$ & \\
\hline Sem complicações & $2(15,4)$ & $1(7,1)$ & 0,074 \\
\hline Óbito, n (\%) & $5(38,5)$ & $7(50,0)$ & 0,598 \\
\hline
\end{tabular}

Dados expressos em médias \pm desvio padrão e n (\%). IRA - insuficiência respiratória aguda; FAF - ferimento por arma de fogo; PCR - parada cardiorrespiratória; AVC - acidente vascular cerebral; FAB - ferimento por arma branca; Min minutos; UTI - unidade de terapia intensiva Incluir na nota de rodapé os testes estatísticos aplicados. 


\section{Discussão}

O principal achado deste estudo foi o fato dos pacientes intubados com a máscara laríngea durante o atendimento pré-hospitalar apresentaram menor de tempo de ventilação mecânica e tempo de internação na UTI na fase de internação hospitalar. Contudo, estes resultados apresentados pelos indivíduos intubados com a máscara laríngea não influenciaram de maneira significativa o desfecho dos mesmos, não apresentando taxa de óbito significativa quando comparado ao grupo intubado com tubo endotraqueal. Tal fato vai de encontro a outro estudo ${ }^{13}$, no qual se verificou que, apesar de dispositivos supraglóticos proporcionarem menor tempo de intubação traqueal, não influenciaram em um melhor índice de sobrevida, nas duas horas de admissão hospitalar, em relação aos pacientes intubados com o tubo endotraqueal.

Wang e colaboradores ${ }^{14}$ compararam a utilização do tubo endotraqueal e da máscara laríngea durante o atendimento pré-hospitalar de pacientes acometidos por parada cardiorrespiratória (PCR). Foram avaliadas em seu estudo sobrevida na alta hospitalar e nas primeiras $24 \mathrm{~h}$ após a mesma, bem como complicações pulmonares e de vias aéreas na alta hospitalar. Os autores encontraram melhores resultados em relação a utilização do tubo endotraqueal em relação a todas as variáveis analisadas.

Em outro estudo, também com pacientes acometidos por PCR, Kang e colaboradores ${ }^{15}$ compararam a utilização do tubo endotraqueal e dispositivos supraglóticos onde observaram que a sobrevida foi maior nos indivíduos que foram intubados com o uso do tubo endotraqueal. Semelhantes resultados também foram encontrados por Benoit e colaboradores ${ }^{16}$, que observaram em sua meta-análise que pacientes intubados com tubo endotraqueal, apresentaram meIhor sobrevida e condição neurológica no momento da admissão hospitalar. No presente estudo, analisamos a taxa de óbito durante a fase de atendimento hospitalar e não observamos diferenças significativas entre os grupos.

Em contrapartida, Kajino e colaboradores ${ }^{11}$ não encontraram diferenças significativas entre a utilização do tubo endotraqueal e dispositivos supraglóticos no atendimento pré-hospitalar ao analisarem a sobrevida no primeiro mês após o atendimento, bem como os desfechos neurológicos. Contudo, relataram ainda que o manejo precoce das vias aéreas está associado aos melhores desfechos. Tal fato corrobora com os nossos achados, tendo em vista que o tempo de atendimento pré-hospitalar foi menor nos indivíduos submetidos a intubação traqueal com a máscara laríngea. Apesar desta variável não ter apresentado diferença significativa, colaborou para melhores desfechos hospitalares como menor tempo de ventilação mecânica e menor tempo de internação na UTI, conforme já mencionado.

Um importante fator a ser ressaltado é que a eficácia do tubo endotraqueal ainda não está clara, uma vez que o nível de treinamento das equipes que prestam $o$ atendimento emergencial pode influenciar no desfecho do processo de intubação ${ }^{13}$. Wang e colaboradores $^{16}$ avaliaram em outro estudo a relação entre a experiência profissional no processo de intubação traqueal e sobrevida após o atendimento pré-hospitalar de causas variadas. Os autores observaram que a experiência por parte da equipe no processo de intubação traqueal está associada a maior sobrevida após o atendimento pré-hospitalar em pacientes acometidos por parada cardíaca. O mesmo não foi observado em pacientes politraumatizados, que é o perfil predominante do presente estudo. Para profissionais com pouca experiência no manejo de vias aéreas, recomenda-se a utilização de dispositivos supraglóticos para procedimentos de intubação traqueal ${ }^{17}$.

Assim como em nosso estudo, a prevalência do sexo masculino no perfil de pacientes atendidos por serviços médicos móveis de emergência, fatores socioculturais específicos de cada região podem justificar a distinção da morbidade entre homens e mulheres $^{19-23}$. Nesse estudo, verificamos também um predomínio de causas relacionadas ao trauma nos atendimentos realizados, o que contrasta com os demais estudos que abordam esse tema11,14,15,16.

Becker e colaboradores ${ }^{24}$ observaram em seu estudo que maiores taxas de mortalidade estavam associadas a pacientes categorizados como alta prioridade na triagem do setor de emergência hospitalar. Acreditamos, então, que o estado de gravidade do indivíduo no momento do atendimento pré-hospitalar pode ser um dos principais fatores que contribuem para desfechos duros, independente do dispositivo utilizado para o manejo das vias aéreas. Entretanto, no presente estudo a gravidade dos pacientes não foi avaliada. 
Apesar dos benefícios observados com a utilização da máscara laríngea nos atendimentos de emergência, não se pode descartar a ocorrência de complicações associadas à sua utilização. Segundo Benhard e colaboradores ${ }^{25}$, os quais avaliaram oito relatos de caso, observou colocação incorreta do referido instrumento na traqueia, deslocamento e/ou colocação incorreta da máscara na faringe, edema de língua e faringe, com subsequentemente dificuldade de laringoscopia, ventilação inadequada devido à obstrução das vias aéreas não reconhecida e pneumotórax hipertensivo. As alterações anteriormente mencionadas não foram observadas em nosso estudo. Contudo, cabe ressaltar que é primordial um treinamento constante dos profissionais de saúde que atuam na área pré-hospitalar para que se refine a perícia dos atendimentos, independente dos dispositivos e técnicas utilizadas durante os mesmos. Este estudo apresenta pontos fortes, como a comparação de duas ferramentas distintas para a intubação no momento pré-hospitalar e o desfecho resultante da utilização de cada uma delas. A limitação deste estudo está relacionada ao tamanho amostral e ao fato de não termos utilizado nenhum instrumento de avaliação de gravidade na população estudada.

\section{Conclusão}

A utilização da máscara laríngea durante o atendimento pré-hospitalar demonstrou ter desfechos positivos sobre o tempo de ventilação mecânica e tempo de internação na UTI na população estudada.

\section{Contribuições dos autores}

Prestes RB participou da concepção, delineamento, coleta de dados de pesquisa, interpretação dos resultados e redação do artigo científico. Fontela PC participou da concepção, delineamento, e interpretação dos resultados. Coutinho WM participou da concepção, delineamento e redação. Forgiarini Junior LA participou da concepção, delineamento, análise estatística e interpretação dos resultados.

\section{Conflitos de interesses}

Nenhum conflito financeiro, legal ou político envolvendo terceiros (governo, empresas e fundações privadas, etc.) foi declarado para nenhum aspecto do trabalho submetido (incluindo mas não limitando-se a subvenções e financiamentos, participação em conselho consultivo, desenho de estudo, preparação de manuscrito, análise estatística, etc.).

\section{Referências}

1. Brasil. Ministério da Saúde. Secretaria de Atenção à Saúde. Portaria GM/MS 2048, de 05 de novembro de 2002. Aprova o regulamento técnico dos sistemas estaduais de urgência e emergência. Diário Oficial da União. 2002 nov. 12; Seção 1. p 56.

2. Sasser S, Varghese M, Kellermann A, Lormand JD. Prehospital trauma care systems. Geneva: World Health Organization; 2005.

3. Wang HE, Lave JR, Sirio CA, Yealy DM. Paramedic intubation errors: isolated events or symptoms of larger problems? Health Aff (Millwood). 2006;25(2):501-9. doi: 10.1377/hlthaff.25.2.501

4. American Society of Anesthesiologists Task Force on Management of the Difficult Airway. Practice guidelines for management of the difficult airway: an updated report by the American Society of Anesthesiologists Task Force on Management of the Difficult Airway. Anesthesiology. 2003;98(5):1269-77. doi: 10.1097/00000542-200305000-00032

5. Henderson JJ, Popat MT, Latto IP, Pearce AC, Difficult Airway Society. Difficult Airway Society guidelines for management of the unanticipated difficult intubation. Anaesthesia. 2004;59(7):675-94. doi: 10.1111/j.1365-2044.2004.03831.x

6. Pedersoli CE, Darli MCB, Silveira RCPC, Chianca TCM, Cyrillo RMZ, Galvão CM. O uso da máscara laríngea pelo enfermeiro na ressuscitação cardiopulmonar: revisão integrativa da literatura. Texto Contexto Enferm. 2011;20(2):376-83. doi: 10.1590/S0104$\underline{07072011000200021}$

7. Polat R, Aydin GB, Ergil J, Savin M, Kokulu T, Oztürk I. Comparação da máscara laríngea i-gel (i-gelTM) com a máscara laríngea clássica (LMA- -ClassicTM) em relação ao desempenho clínico. Rev Bras Anestesiol. 2015;65(5):343-8. doi: 10.1016/j. bjan.2014.02.009

8. Siddiqui S, Seet E, Chan WY. The use of laryngeal mask airway Supreme ${ }^{\mathrm{TM}}$ in rescue airway situation in the crítical care unit. Singapore Med J. 2014;55(12):e205-6. doi: 10.11622/ smedj.2014187 
9. Thomaz RR, Whitaker IY. Uso da máscara laríngea em pacientes com parada cardiorrespiratória: revisão sistemática. Rev Eletr Enf. 2013;15(3):808-15. doi: 10.5216/ree.v15i3.20453

10. Wnent J, Franz R, Seewalds S, Lefering R, Fischer M, Bohn A et al. Difficult intubation and outcome after out-of-hospital cardiac arrest: a registry-based analysis. Scand J Trauma Resusc Emerg Med. 2015;23:43. doi: 10.1186/s13049-015-0124-0

11. Kajino K, Iwami T, Kitamura T, Daya M, Ong ME, Nishiuchi T et al. Comparison of supraglottic airway versus endotracheal intubation for the pre-hospital treatment of out-of-hospital cardiac arrest. Crit Care. 2011;15(5):R236. doi: 10.1186/cc10483

12. Ott T, Fischer M, Limbach T, Schmidtmann I, Piepho T, Noppens RR. The novel intubating laryngeal tube (iLTS-D) is comparable to the intubating laryngeal mask (Fastrach) - a prospective randomised manikin study. Scand J Trauma Resusc Emerg Med. 2015;23:44. doi: 10.1186/s13049-015-0126-y

13. Fan YJ, Dai CY, Huang DC, Wang ML. Does tracheal intubation really matter? Discrepant survival between laryngeal mask and endotracheal intubation during out-of-hospital cardiac arrest. J Formos Med Assoc. 2017;116(2):134-35. doi: 10.1016/j. jfma.2016.11.001

14. Wang HE, Szydlo D, Stouffer JÁ, Lin S, Carlson JN, Vaillancourt C et al. Endotracheal intubation versus supraglottic airway insertion in out-of-hospital cardiac arrest. Resuscitation. 2012;83(9):1061-6. doi: 10.1016/j.resuscitation.2012.05.018

15. Kang K, Kim T, Ro YS, Kim YJ, Song KJ, Shin SD. Prehospital endotracheal intubation and survival after out-of-hospital cardiac arrest: results from the Korean nationwide registry. Am J Emerg Med. 2016;34(2):128-32. doi: 10.1016/j.ajem.2015.09.036

16. Benoit JL, Gerecht RB, Steuerwald MT, McMullan JT. Endotracheal intubation versus supraglottic airway placement in out-of-hospital cardiac arrest: A meta-analysis. Resuscitation 2015;93:20-6. doi: 10.1016/j.resuscitation.2015.05.007

17. Wang HE, Balasubramani GK, Cook LJ, Lave JR, Yealy DM. Out-of-hospital endotracheal intubation experience and patient outcomes. Ann Emerg Med. 2010;55(6):527-37. doi: 10.1016/j. annemergmed.2009.12.020

18. Gruber C, Nabecker S, Wohlfarth P, Ruetzler A, Roth D, Kimberger $O$ et al. Evaluation of airway management associated hands-off time during cardiopulmonary resuscitation: arandomised manikin follow-up study. Scand J Trauma Resusc Emerg Med. 2013;21:10. doi: 10.1186/1757-7241-21-10
19. Kopsel ME, Vicensi MC. Perfil epidemiológico e demográfico dos usuários do serviço de emergência do Hospital Universitário Santa Terezinha atendidos pelo convênio SUS. Unoesc \& Ciência ACBS. 2012;3(2):131-42.

20. Magnago TSBS, Rosa TP, Tavares JP, Lima SBS, Schimidt MD, Silva RM. Perfil dos pacientes atendidos a sala de emergência do pronto socorro de um Hospital Universitário. R Enferm UFSM. 2011;1(1):51-60. doi: 10.5902/217976922090

21. Andrade SSCA, Sá NNB, Carvalho MGO, Lima CM, Silva MMA, Moraes Neto $\mathrm{OL}$ et al. Perfil das vítimas de violências e acidentes atendidas em serviços de urgência e emergência selecionados em capitais brasileiras: vigilância de Violências e acidentes, 2009. Epidemiol Serv Saúde. 2012;21(1):21-30. doi: 10.5123/S1679$\underline{49742012000100003}$

22. Gonsaga RAT, Brugugnolli ID, Zanutto TA, Gilioli JP, Silva LFC, Fraga GP. Características dos atendimentos realizados pelo serviço de atendimento móvel de urgência no município de Catanduva, estado de São Paulo, Brasil, 2006 a 2012. Epidemiol Serv Saúde. 2013;22(2):317-24. doi: 10.5123/S167949742013000200013

23. Rodrigues AIG, Korinfsky JP, Santos ADB, Oliveira ANS, Almeida LR, Moura LA. Perfil dos usuários atendidos no serviço de emergência em um hospital universitário em Pernambuco. Rev Baiana Saúde Pública. 2015;39(1):13-24.

24. Becker JB, Lopes MCBT, Pinto MF, Campanharo CRV, Barbosa DA, Batista REA. Triagem no serviço de emergência: associação entre as suas categorias e os desfechos do paciente. Rev Esc Enferm USP. 2015;49(5):783-89. doi: 10.1590/S0080$\underline{623420150000500011}$

25. Bernhard M, Beres W, Timmermann A, Stepan R, Greim $C A$, Kaisers UX et al. Prehospital airway management using the laryngeal tube. An emergency department point of view. Anaesthesist. 2014;63(7):589-96. doi: 10.1007/s00101-014-2348-1 